\title{
A 2-D Discrete Cubic Chaotic Mapping with Symmetry
}

\section{Una cartografía caótica cúbica discreta con simetría}

DOI: $10.46932 / \mathrm{sfjdv2n4-012}$

Received in: May 1st, 2021

Accepted in: Jun 30th, 2021

\begin{abstract}
M. Mammeri
Department of Mathematics, Faculty of Mathematics and science of Matter, and Department of Biological Sciences, Faculty of Nature and Life Sciences, Kasdi Merbah University, Ouargla, Algeria E-mail: mammeri_muh@yahoo.fr.
\end{abstract}

\begin{abstract}
In the theoretical research of chaotic dynamical system, the different type of bifurcations is a very interesting powerful tool for analyzing the qualitative behavior of chaotic dynamical system; this short paper is devoted to analysis of a simple 2-D symmetry discrete chaotic map with quadratic and cubic nonlinearities. The dynamical behaviors of the map are investigated by mathematical analysis and simulated numerically using package of Matlab. We compute numerically the bifurcation diagram and largest Lyapunov exponent and phase portraits. The research results indicate that there are interesting nonlinear physical phenomena in this simple 2-D symmetry discrete cubic map, such as symmetry bifurcation, Hopf bifurcation, symmetry breaking bifurcation and identical symmetric attractors. The important nonlinear physical phenomena obtained in this paper would benefit the study of the cubic chaotic map and the development of the theory of chaotic discrete dynamical systems.
\end{abstract}

Keywords: 2-D symmetry map, cubic map, symmetry-breaking bifurcation, Hopf bifurcation, symmetric attractors.

\section{RESUMEN}

En la investigación teórica de los sistemas dinámicos caóticos, los diferentes tipos de bifurcaciones son una herramienta poderosa muy interesante para analizar el comportamiento cualitativo de los sistemas dinámicos caóticos; este breve artículo está dedicado al análisis de un mapa caótico discreto de simetría bidimensional simple con no linealidades cuadráticas y cúbicas. Los comportamientos dinámicos del mapa se investigan mediante análisis matemático y se simulan numéricamente utilizando el paquete de Matlab . Calculamos numéricamente el diagrama de bifurcación y el mayor exponente de Lyapunov y los retratos de fase. Los resultados de la investigación indican que existen interesantes fenómenos físicos no lineales en este sencillo mapa cúbico discreto de simetría 2-D, como la bifurcación de simetría, la bifurcación de Hopf, la bifurcación de ruptura de simetría y los atractores simétricos idénticos. Los importantes fenómenos físicos no lineales obtenidos en este trabajo beneficiarían el estudio del mapa cúbico caótico y el desarrollo de la teoría de los sistemas dinámicos discretos caóticos.

Palabras clave: Mapa de simetría bidimensional, mapa cúbico, bifurcación de ruptura de simetría, bifurcación de Hopf, atractores simétricos. 


\section{INTRODUCTION}

Many papers have described a class of systems ${ }^{1-2}$. The study of chaotic behaviour of discrete mapping is a very interesting branch in dynamical systems theory because a large number of science technology and applied science researches such as physics and engineering ${ }^{3-4-5-6-7}$, medicine ${ }^{8}$, industry ${ }^{9}$ systems have been displayed by model of discrete mappings. Moreover however, in the theoretical research of chaotic discrete mapping, the different type of bifurcations are a very interesting powerful tools for analyzing the qualitative behaviour of chaotic discrete mapping, such as period-doubling bifurcation ${ }^{10}$, border collision bifurcation ${ }^{11-12-13}$, border collision period-doubling bifurcation ${ }^{14}$, quasiperiodic bifurcation ${ }^{15-16-17-18}$, Intermittency bifurcation ${ }^{19-20,}$ symmetry-breaking bifurcation ${ }^{5-21-22}$, symmetry-breaking period-doubling bifurcation ${ }^{23}$.

This paper has reported a further investigation into a two-dimensional discrete chaotic map with symmetry capable of generating identical symmetric chaotic attractors from different initial conditions and through different map parameters values. Dynamical behaviour has been reported within some map parameter values range. The essential dynamical behaviours, including largest Lyapunov exponents, bifurcation analysis and phase portraits are also simulated when the map parameter varied to verify map behaviours.

\section{THE 2-D DISCRTE CHAOTIC MAPPING}

The new two-dimensional discrete map (1) is defined by bi-nonlinearity coordinates a function of one variables $y$ and a function of two variables $x$ and $y$. The 2-D discrete map (1) can be described by the following equation:

$$
\left(\begin{array}{l}
\bar{x} \\
\bar{y}
\end{array}\right)=\left(\begin{array}{c}
y^{2} \\
a y-a x^{2} y
\end{array}\right)
$$

Where $x$ and $y$ are the state variables of the map, and $a$ is the map parameter. For $a=0$, the map (1) reduces to a two-dimensional discrete linear map. The map (1) is defined for all points in the plane, the associated quadratic $f(x, y)=y^{2}$ and cubic $g(x, y)=a y-a x^{2} y$ bi-nonlinear functions are of class $C^{\infty}\left(\mathbb{R}^{2}\right)$ and the map (1) is symmetric under the coordinate transformation $(x, y) \rightarrow(-x, y)$. Furthermore, the map (1) belongs to the general form of the two-dimensional cubic map given by:

$$
\left(\begin{array}{l}
\bar{x} \\
\bar{y}
\end{array}\right)=
$$




$$
\left(\begin{array}{l}
a_{0}+a_{1} x+a_{2} y+a_{3} x^{2}+a_{4} y^{2}+a_{5} x y+a_{6} x^{2} y+a_{7} x y^{2}+a_{8} x^{3}+a_{9} y^{3} \\
b_{0}+b_{1} x+b_{2} y+b_{3} x^{2}+b_{4} y^{2}+b_{5} x y+b_{6} x^{2} y+b_{7} x y^{2}+b_{8} x^{3}+b_{9} y^{3}
\end{array}\right)
$$

Where $(x, y) \in \mathbb{R}^{2}$ and $\left(a_{i}, b_{i}\right)_{0 \leq i \leq 9} \in \mathbb{R}^{20}$ are map parameter.

\section{STABILITY RESULTS}

Briefly, the fixed points of map (1) are:

$(0,0)$ and $\left(\sqrt{\frac{a-1}{a}}, \pm \sqrt[4]{\frac{a-1}{a}}\right)$

and the Jacobi matrix of the map (1) evaluated at the fixed point $(x, y)$ is:

$$
J_{(x, y)}=\left(\begin{array}{cc}
0 & 2 y \\
-2 a x y & a-x^{2}
\end{array}\right)
$$

The characteristic polynomial of the Jacobi matrix $J_{(x, y)}$ is:

$$
P(\lambda)=\lambda^{2}-a\left(1-x^{2}\right) \lambda+4 a x y^{2}
$$

By criteria $\mathrm{in}^{24}$, the trivial fixed point $(0,0)$ of the map (1) is asymptotically stable if and only if the following conditions hold:

$$
1-a>0,1+a>0
$$

or, equivalently,

$$
-1<a<1
$$

For example, if we choose $a=0.2$ and $(0.01,0.01)$ the fixed point $(0,0)$ is asymptotically stable, and we have the following two eigenvalues $\lambda_{1}=0$ and $\lambda_{2}=0.2$ thus $\left|\lambda_{i(1 \leq i \leq 2)}\right|<1$ (see Fig. 1a).

And if we choose $a=0.7$ and $(0.01,0.01)$ the fixed point $(0,0)$ is asymptotically stable, and we have the following two eigenvalues $\lambda_{1}=0$ and $\lambda_{2}=0.7$ thus $\left|\lambda_{i(1 \leq i \leq 2)}\right|<1$ (see Fig. 1b). 
Fig. 1a: Time series $(x, y)$ with $(0.01,0.01)$ and $a=0.20$.

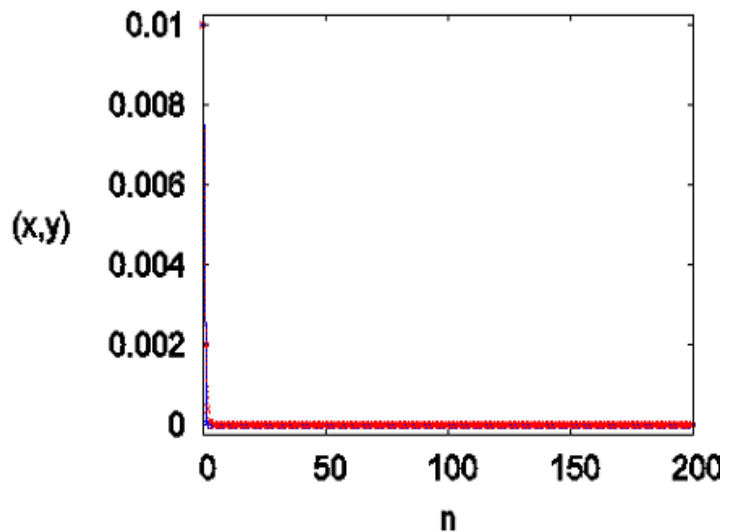

Fig. 1b: Time series $(x, y)$ with $(0.01,0.01)$ and $a=0.70$.

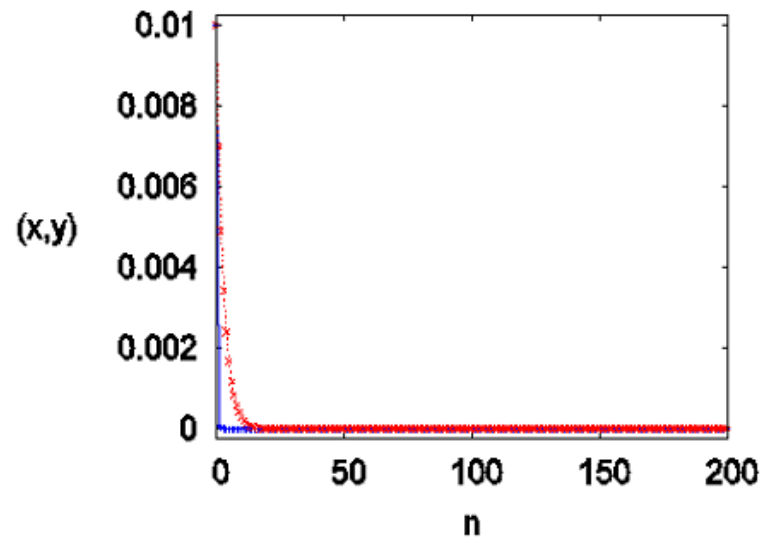

On the other hand, the local stability of $(0,0)$ is studied by evaluating the of the Jacobi $J_{(0,0)}$. Than one has the following results:

(a) $\left|\lambda_{2}\right|<1$, if and only if $-1<a<1$, map (1) is attracting at this fixed point.

(b) $\left|\lambda_{2}\right|>1$, if and only if $a \in(-\infty,-1) \cup(1,+\infty)$, map (1) is a saddle at this fixed point.

By criteria $i^{24}$, the fixed points $\left(\sqrt{\frac{a-1}{a}}, \pm \sqrt[4]{\frac{a-1}{a}}\right)$ of the map (1) are asymptotically stable if and only if the following conditions hold:

$$
\begin{gathered}
1-1+4(a-1)>0 \\
1+1+4(a-1)>0 \\
1-4(a-1)>0
\end{gathered}
$$


or, equivalently,

$$
1<a<\frac{5}{4}
$$

For example, if we choose $a=1.05$ and $(0.01,0.01)$ then with this value the fixed points $(0.21822, \pm 0.46714)$ are asymptotically stable, and we have the following two eigenvalues $\lambda_{1}=$ 0.27639 and $\lambda_{2}=0.72361$ thus $\left|\lambda_{i(1 \leq i \leq 2)}\right|<1$ (see Fig. 2a).

Fig. 2a: Times series $(x, y)$ with $(0.01,0.01)$ and $a=1.20$.

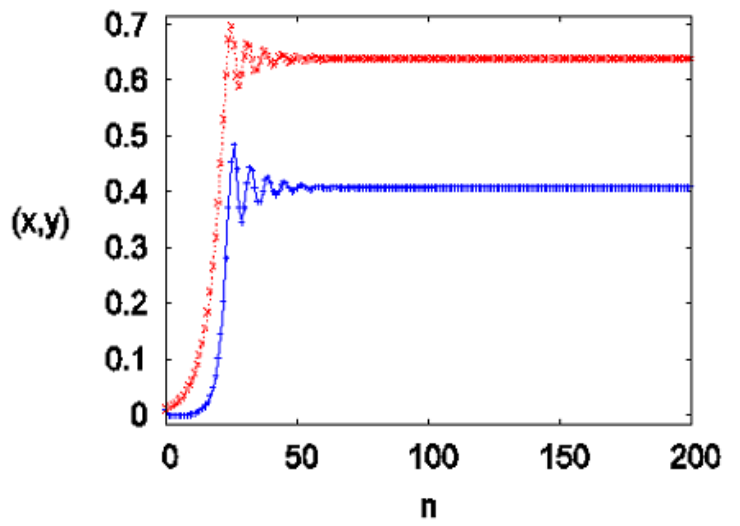

Fig. 2b: Times series $(x, y)$ with $(0.01,0.01)$ and $a=1.05$.

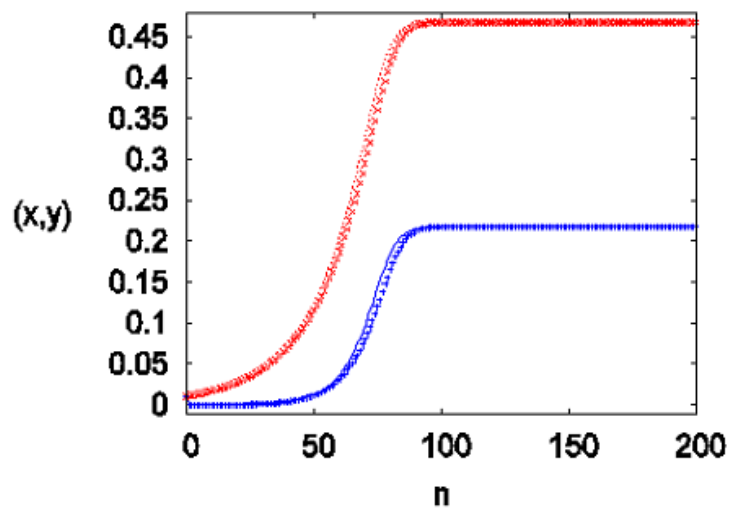

On the other hand, the local stability of points $\left(\sqrt{\frac{a-1}{a}}, \pm \sqrt[4]{\frac{a-1}{a}}\right)$ is studied by evaluating the eigenvalues of the Jacobi $J\left(\sqrt{\frac{a-1}{a}}, \pm \sqrt[4]{\frac{a-1}{a}}\right)$.

If $a \in(-\infty, 0) \cup\left(1, \frac{17}{16}\right)$ the eigenvalues of $J\left(\sqrt{\frac{a-1}{a}}, \pm \sqrt[4]{\frac{a-1}{a}}\right)$ are: 
$\lambda_{1}=\frac{1-\sqrt{17-16 a}}{2}$ and $\lambda_{2}=\frac{1+\sqrt{17-16 a}}{2}$.

Than one have the following results:

(a) $\left|\lambda_{1}\right|<1$ and $\left|\lambda_{2}\right|<1$ if and only if $1<a<\frac{17}{16}$, map (1) is attracting at this fixed point.

(b) $\lambda_{1}=\lambda_{2}=\frac{1}{2}<1$ if and only if $a=\frac{17}{16}$, map (1) is attracting at this fixed point.

(c) $\left|\lambda_{1}\right|>1$ and $\left|\lambda_{2}\right|<1$ or $\left(\left|\lambda_{1}\right|<1\right.$ and $\left.\left|\lambda_{2}\right|>1\right)$, impossible, map (1) is not a saddle at this fixed point.

(d) $\left|\lambda_{1}\right|>1$ and $\left|\lambda_{2}\right|>1$, if and only $a<0$, map (1) is repelling at this fixed point.

If $a>\frac{17}{16}$ the eigenvalues of $J\left(\sqrt{\frac{a-1}{a}}, \pm \sqrt[4]{\frac{a-1}{a}}\right)$ are:

$\lambda_{1}=\frac{1-i \sqrt{-(17-16 a)}}{2}$ and $\lambda_{2}=\frac{1+i \sqrt{-(17-16 a)}}{2}$.

Than one have the following results:

(a) $\left|\lambda_{1}\right|<1$ and $\left|\lambda_{2}\right|<1$, if and only if $\frac{17}{16}<a<\frac{5}{4}$, map (1) is attracting at this fixed point.

(b) $\lambda_{1}=\lambda_{2}=\frac{1}{2}<1$ if and only if $a=\frac{17}{16}$, map (1) is attracting at this fixed point.

(c) $\left|\lambda_{1}\right|>1$ and $\left|\lambda_{2}\right|<1$ or $\left(\left|\lambda_{1}\right|<1\right.$ and $\left.\left|\lambda_{2}\right|>1\right)$, impossible, map (1) is not a saddle at this fixed point.

(c) $\left|\lambda_{1}\right|>1$ and $\left|\lambda_{2}\right|>1$, if and only $a>\frac{5}{4}$, map (1) is repelling at this fixed point.

\section{SYMMERTY BIFURCATION AND ATTRACTORS}

In this section, the dynamical behaviors of the map are investigated and simulated numerically. There are several possible ways for a discrete map to make a transition from regular behaviour to chaotic behaviour. Bifurcation diagrams display these routes to determine the behaviour and chaotic zones, we compute numerically the bifurcation diagram and largest Lyapunov exponent in the range $[-1.52,1.52]$. Figures. 3 shows respectively, the symmetric bifurcation diagram and the symmetric Lyapunov exponent spectrum diagram of map (1). Figures 4 illustrate some observed of identical attractors and chaotic attractors generated by two symmetric $\pm(0.01,0.01)$ initial conditions and within two symmetrical $\pm a$ map parameters values. All figures show that the attractors are symmetric about the y-axis. The map (1) exhibits the following dynamical behaviors (see Fig. 3a and Fig. 3b):

(a) $-1.52 \leq a<-1.25$, map (1) is chaotic (LEs $>0$, see Fig. 4d, Fig. 4e and Fig. 4f), periodic windows exists in the chaotic zone (LEs $\leq 0$, see Fig. 4a, Fig. $4 \mathrm{~b}$ and Fig. $4 \mathrm{c}$ ).

(b) $a=-1.25$, the Hopf bifurcation occurs in the map (1) ( see Fig. 3a). 
(c) $-1.25<a<-1$, the map (1) is in steady state (LEs $<0$, see Fig. 3a).

(d) $a=-1$, the symmetry-breaking bifurcation occurs at this point and the first branch occurs with the two symmetrical $\pm(0.01,0.01)$ initial conditions (see Fig. 3a).

(e) $-1<a<1$, map (1) is stable (LEs $<0$, see Fig. 3a).

(f) $a=1$, the symmetry-breaking bifurcation occurs at this point and the second branch occurs with the two symmetrical $\pm(0.01,0.01)$ initial conditions (see Fig. 3a).

(g) $1<a<1.25$, the map (1) is in steady state (LEs $<0$, see Fig. 3a).

(h) $a=1.25$, the Hopf bifurcation occurs in the map (1) (see Fig. 3a).

(k) $1.25<a \leq 1.52$, map (1) is chaotic (LEs >0, see Fig. 4d, Fig. 4e and Fig. 4f ), periodic windows exists in the chaotic zone (LEs $\leq 0$, see Fig. 4a, Fig. 4b and Fig. 4c).

Fig. 3a: Bifurcation diagram with $\pm(0.01,0.01)$ and $a \in[-1.52,1.52]$.

(a)

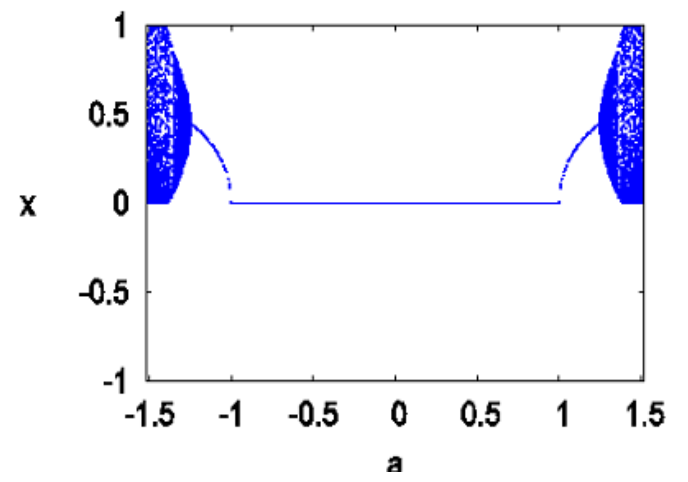

Fig. 3b: Lyapunov exponent with $\pm(0.01,0.01)$ and $a \in[-1.52,1.52]$.

(b)

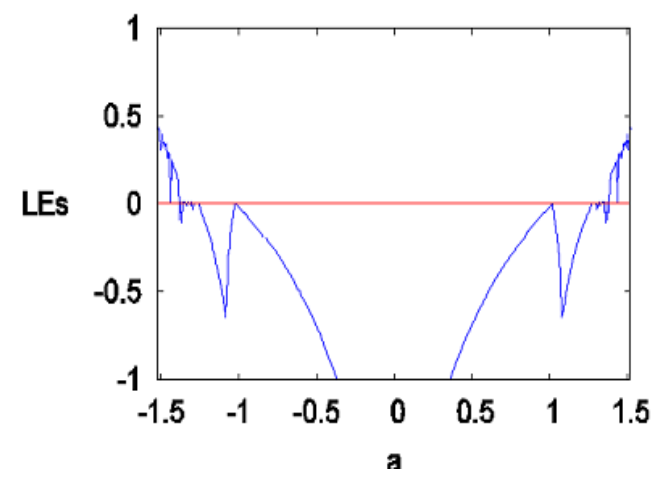


Fig. 4a: Attractor with $\pm(0.01,0.01)$ and $a \pm 1.41$.

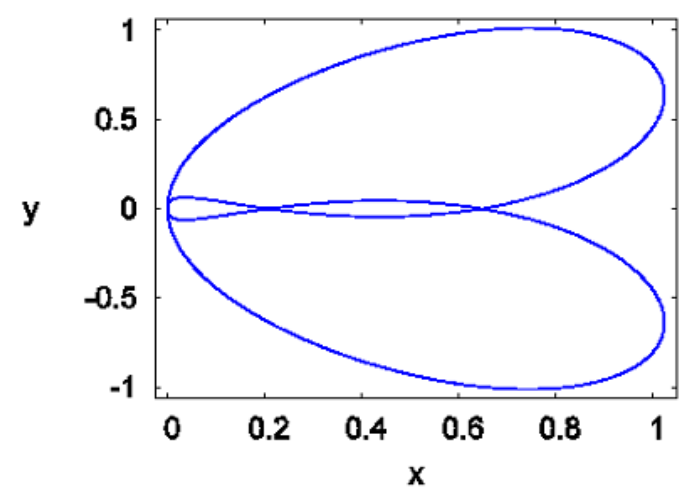

Fig. 4b: Attractor with $\pm(0.01,0.01)$ and $a \pm 1.43$.

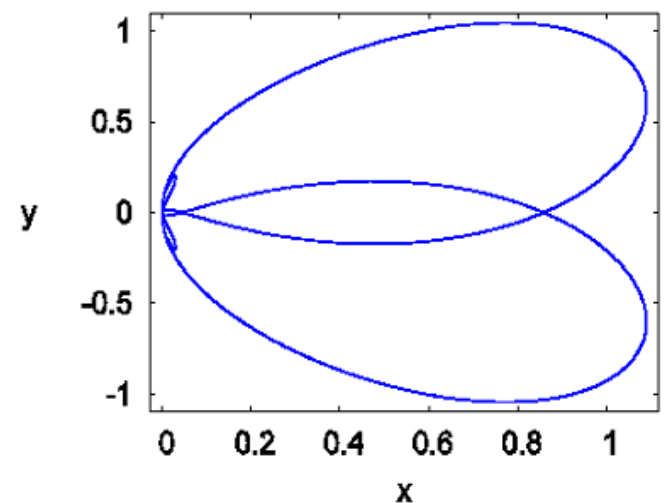

Fig. 4c: Attractor with $\pm(0.01,0.01)$ and $a \pm 1.45$.

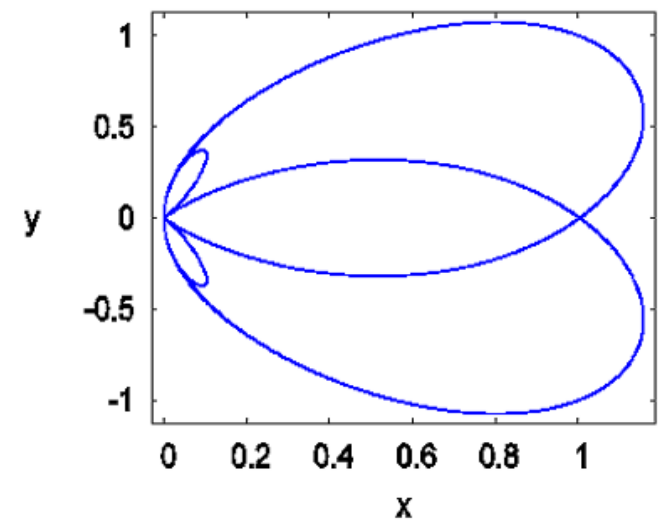

Fig. 4d: Attractor chaotic with $\pm(0.01,0.01)$ and $a \pm 1.47$.

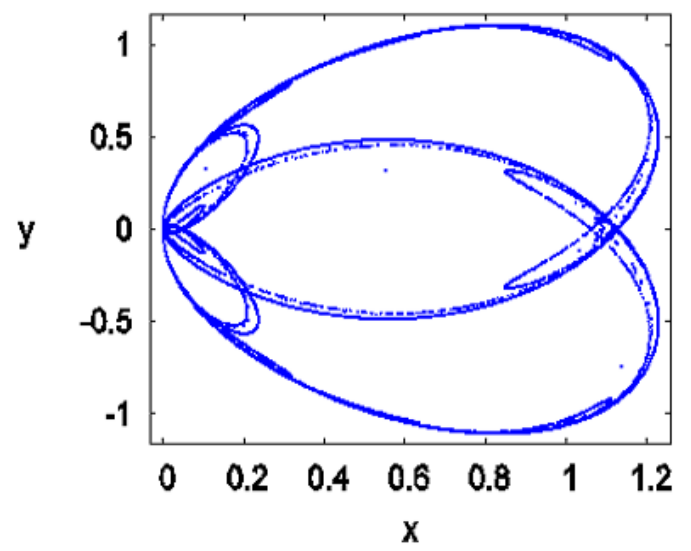


Fig. 4e: Attractor chaotic with $\pm(0.01,0.01)$ and $a \pm 1.49$.

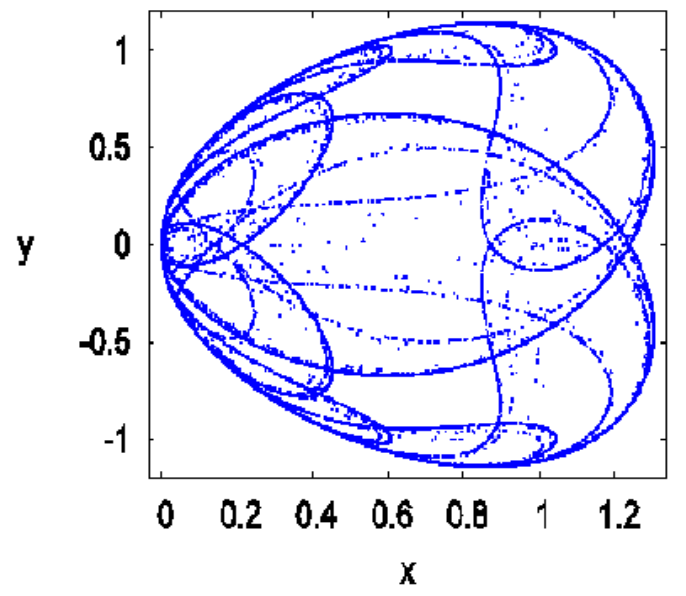

Fig. 4f: Attractor chaotic with $\pm(0.01,0.01)$ and $a \pm 1.51$.

\section{CONCLUSION}

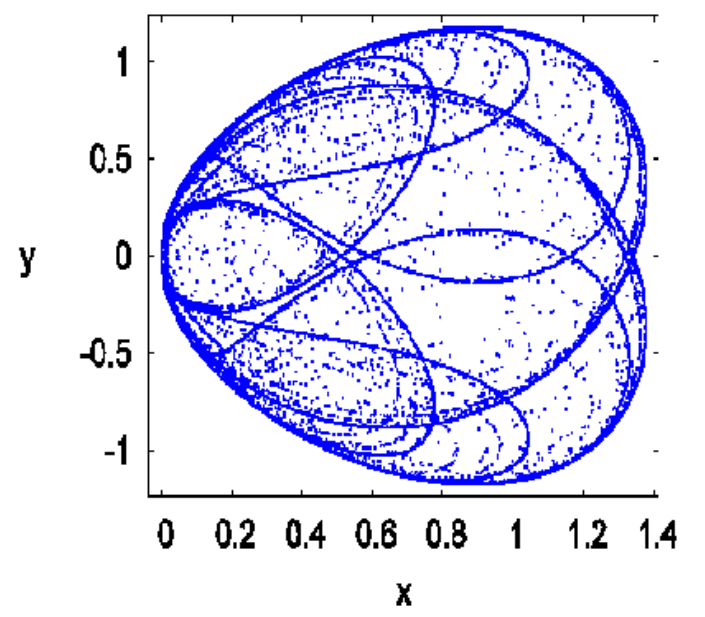

This paper has presented a further investigation into a two-dimensional discrete chaotic cubic mapping generating symmetric chaotic attractors. Dynamical behaviour has been reported within some values map parameter range. The basic dynamical behaviours, including stability, evolution of attractors, Lyapunov exponent spectrum, bifurcation analysis and phases portrait are also illustrated to verify map behaviours.

\section{SIGNIFICANCE OF STUDY}

The study of 2-D discrete chaotic cubic mapping such as with symmetry is interesting contribution to the development of the theory of dynamical systems. The important nonlinear physical phenomena obtained in this paper would benefit the study of the cubic chaotic mapping and the development of the theory of chaotic systems.

\section{REFERENCES}


[1] María José Garrido Antón., 2021. Valuation of Subject Identity by Indirect Personality Profiling (V0) in the System for Analysis of Validity in Evaluation (SAVE), South Florida Journal of Development, 2(2), 3697-3699.

[2] Yury I. Lobanovsky., 2021. Classification is the Method of System Problems Detection, South Florida Journal of Development. 2(3), 3879-3889.

[3] Benerjee S and Verghese GC., 2001. Nonlinear phenomena in power electronics, attractor, bifurcations. IEEE Press, Piscataway, USA.

[4] Luo, A.C.J., 2012. Discrete and switching dynamical systems. Higher Education Press, Beijing.

[5] K. Mandal, S. Banerjee, C. Chakraborty., 2013. Symmetry-Breaking Bifurcation in Series-Parallel Load Resonant DC-DC Converters. IEEE Trans. Circuits Syst. I., vol.60, no.3, 778-787.

[6] Banerjee S, Parui S and Gupta A., 2004. Dynamical Effects of Missed Switching in Current-Mode Controlled dc-dc Converters. IEEE Trans. Circuits \& Systems-II 51 649-54.

[7] Gałach M., 2003. Dynamics of the tumor-immune system competition-the effect of time delay. International Journal of Applied Mathematics and Computer Science, 13: 395-406.

[8] Bischi GI and Tramontana F ., 2010. Three-dimensional discrete-time Lotka-Volterra models with an application to industrial cluster". Communications in Nonlinear Science and Numerical Simulation, 15(10): 3000-3014.

[9] Hénon. M., 1976. A two-dimensional mapping with a strange attractor. Communications in Mathematical Physics, 50(1): 69-77.

[10] Lozi. R., 1978. Un attracteur étrange du type attracteur de Hénon, Journal de Physique Colloque C5, Supplèment au $n^{0} 8,39,9-10$.

[11] G. H. Yuan, S. Banerjee, E. Ott, and J. A. Yorke.,1998. Border collision bifurcations in the buck converter, IEEE Trans. Circuits Systems-I,45, 707-716.

[12] I. Sushko and L. Gardini., 2006. Center bifurcation for a two-dimensional piecewise linear map. In T. Puu and I. Sushko, editors, Business Cycle Dynamics: Models and Tools., pages 49-78. SpringerVerlag, New York.

[13] E. Zeraoulia., 2005. A new chaotic map attractor from 2-D discrete mapping via border collision period-doubling bifurcation scenario, Discrete Dynamics in Nature and Society, vol 2005, 235-238.

[14] E. Zeraoulia, J.C Sprott., 2008. A minimal 2-D quadratic map with quasi-periodic route to chaos, International Journal of Bifurcation and Chaos, Vol. 18, N o. 5, 1567-1577.

[15] M. Mammeri., 2020. Dynamical properties of a 2-D non-invertible system, International Journal of Advanced and Applied Sciences, Vol. 7, no. 5, 98-18.

[16] M. Mammeri., 2017. Symmetry and Periodic-Chaos in 3-D Sinusoid Discrete Maps, Bulletin of Mathematical Analysis and Applications, Vol. 9.no. 1, 1-9. 
[17] M. Mammeri., 2018. Precise long-term prediction of behavior in a 3-D chaotic map, Journal of Engineering and Applied Sciences, Vol. 13, no. 13, 4859-4865.

[18] Ciliberto, S. and Bigazzi, P., 1988. Spatiotemporal Intermittency in Rayleigh-B enard Convection, Phys. Rev. Lett., 60, 286-289.

[19] E. Ott., 1993. Chaos in dynamical systems, Cambridge University Press.

[20] K. Mandal, S. Banerjee, C. Chakraborty., 2011. Symmetry-breaking bifurcation in load resonant dc-dc converters, in Proc. International Symposium on Circuits and Systems, pp.1327-1330.

[21] K. Mandal, S. Banerjee, C. Chakraborty, "Symmetry-Breaking Bifurcation in Series-Parallel Load Resonant DC-DC Converters”, IEEE Trans. Circuits Syst. I, vol.60, no.3, pp.778-787, Mar, 2013

[22] H. Zhang, Y.-D. Chu, W.-C. Ding, et al., 2013. Bifurcation control of a cubic symmetry discrete chaotic system, Acta Phys. Sin., vol. 62, no. 4, pp. 040202.

[23] Elaydi S.,1996. An introduction to difference equations. Springer, Berlin, Germany. 